La Revue

des Droits

de l'Homme

\section{La Revue des droits de l'homme}

Revue du Centre de recherches et d'études sur les droits fondamentaux

Actualités Droits-Libertés | 2014

\title{
Précisions sur les droits de la Charte sociale européenne bénéficiant aux étrangers en situation irrégulière
}

Droits sociaux et droits des étrangers (CEDS)

\section{Carole Nivard}

\section{OpenEdition}

Journals

Édition électronique

URL : http://journals.openedition.org/revdh/982

DOI : $10.4000 /$ revdh.982

ISSN : 2264-119X

Éditeur

Centre de recherches et d'études sur les droits fondamentaux

Référence électronique

Carole Nivard, « Précisions sur les droits de la Charte sociale européenne bénéficiant aux étrangers en situation irrégulière », La Revue des droits de l'homme [En ligne], Actualités Droits-Libertés, mis en ligne le 27 novembre 2014, consulté le 19 avril 2019. URL : http://journals.openedition.org/revdh/982 ; DOI : $10.4000 /$ revdh. 982

Ce document a été généré automatiquement le 19 avril 2019.

Tous droits réservés 


\section{Précisions sur les droits de la Charte sociale européenne bénéficiant aux étrangers en situation irrégulière}

Droits sociaux et droits des étrangers (CEDS)

\section{Carole Nivard}

1 Deux décisions sur le bien-fondé rendues publiques le 10 novembre dernier apportent d'importantes confirmations et précisions quant aux droits de la charte sociale européenne dont bénéficient les étrangers même en situation irrégulière. Il s'agit des décisions Conférence des Eglises européennes (CEC) C. Pays-Bas, n 90/2013 et Fédération européenne des Associations nationales travaillant avec les Sans-abri (FEANTSA) c. Pays Bas, $n^{\circ} 86 / 2012$ rendues respectivement les $1^{\text {er }}$ et 2 juillet 2014 par le Comité européen des droits sociaux (CEDS).

2 Dans la première affaire, l'organisation réclamante ${ }^{1}$ invoque l'inconventionnalité du droit et de la pratique aux Pays-Bas qui excluent du bénéfice de l'assistance sociale d'urgence, notamment du droit d'obtenir des vivres, une solution d'hébergement et des vêtements, les étrangers adultes en situation irrégulière. Dans la seconde affaire, l'organisation FEANTSA allègue l'absence de conformité de la situation nationale à la Charte sociale du fait de l'existence de critères restrictifs à l'accès aux centres d'hébergement d'urgence, ainsi que de l'insuffisance quantitative et qualitative de ces centres qui portent atteinte aux droits des sans-abri ressortissants néerlandais mais également à ceux des étrangers qu'ils soient ou non en situation irrégulière.

3 Si le fond des allégations ne se recoupent pas complètement, les décisions ont été rendues lors d'une même session du Comité et peuvent être analysées ensemble dans la mesure où elles traitent toutes deux la question de l'applicabilité de la Charte sociale européenne aux étrangers en situation irrégulière ainsi que du droit à une assistance sociale d'urgence y compris un hébergement. 
Comité d'adresser pour la première fois des mesures immédiates au Gouvernement ${ }^{2}$. Le Règlement intérieur du Comité prévoit en effet que la Comité peut adopter de telles mesures incitatives "afin d'éviter un risque de dommage grave irréparable et d'assurer le respect effectif des droits reconnus dans la Charte ». Aussi, avant même l'appréciation au fond, le Comité a demandé au Gouvernement défendeur, afin qu'il ne soit pas porté atteinte « $\grave{a}$ l'intégrité des personnes exposées à un risque imminent de dénuement ", de faire "en sorte que leurs besoins essentiels ((logement, habillement, nourriture) soient satisfaits ».

5 Dans ses décisions sur le bien-fondé, le Comité examine la compatibilité de la situation nationale avec principalement l'article $31 \$ 2$ de la Charte sociale révisée, qui engage les Etats à réduire l'état de sans-abri ${ }^{3}$ et l'article $13 \$ 4$ de la Charte qui les engage à assurer un droit à l'assistance sociale et médicale aux ressortissants des autres Etats parties sur un pied d'égalité avec leurs nationaux ${ }^{4}$. Un tel contrôle suppose au préalable d'admettre l'extension du champ d'application de la Charte aux étrangers en situation irrégulière $\left(1^{\circ}\right.$ ), puis de définir le contenu du droit à une assistance sociale d'urgence à laquelle ils peuvent prétendre sur le fondement de ce traité $\left(2^{\circ}\right)$.

\section{$1 \%$ L'extension du champ d'application de la Charte sociale européenne aux étrangers en situation irrégulière}

6 Afin d'admettre l'applicabilité de la Charte aux étrangers en situation irrégulière, le Comité se livre à une interprétation contra legem du traité (A). Il cantonne cependant cette applicabilité à un nombre limité de droits apparaissant comme les plus fondamentaux (B).

\section{A - Une extension contra legem}

7 Les deux réclamations soulèvent la question de la conventionnalité de la situation des migrants en situation irrégulière aux Pays-Bas qui voient leur accès à une assistance sociale, en particulier à un hébergement, refusé. Cette situation serait contraire aux articles $13 \$ 4$ (Assistance d'urgence spécifique aux non-résidents) et $31 \$ 2$ (Réduction de l'état de sans-abri).

Or, admettre l'applicabilité de la Charte sociale européenne aux étrangers en situation irrégulière revient à contredire les termes-mêmes du traité. Le premier paragraphe de l'Annexe à la Charte pose en effet une limitation ratione personae de son champ d'application en disposant que "Sous réserve des dispositions de l'article 12, paragraphe 4, et de l'article 13, paragraphe 4, les personnes visées aux articles $1^{\text {er }} \grave{a} 17$ ne comprennent les étrangers que dans la mesure où ils sont des ressortissants des autres Parties contractantes résidant légalement ou travaillant régulièrement sur le territoire de la Partie contractante intéressée ». L'article 13\$4, invoqué en l'espèce, constitue bien une exception dans la mesure où il bénéficie « aux ressortissants des autres Parties se trouvant légalement sur leur territoire ", donc sans condition de résidence ou de travail.

Reste que le champ d'application personnel de la Charte se trouve bien doublement restreint s'agissant des étrangers : elle ne peut bénéficier ni aux ressortissants d'Etats qui ne sont pas parties à la Charte, ni à l'ensemble des migrants se trouvant en situation irrégulière. Cette limitation particulièrement choquante en matière de droits de 
l'homme n'est toutefois guère étonnante s'agissant de droits sociaux ${ }^{5}$. Elle correspond en tout état de cause à une volonté claire des Etats parties à la Charte de ne pas se voir contraints d'ouvrir aux non-nationaux ou aux non-résidents, l'accès aux politiques sociales offertes à leurs ressortissants. Cette volonté a été confirmée par le maintien de la restriction ratione personae pour la Charte sociale révisée en 1996.

Or, passant outre la lettre du traité, le Comité européen des droits sociaux a admis l'applicabilité de certains droits de la charte aux étrangers, même en situation irrégulière, dans une première décision FIDH c. France ${ }^{6}$ rendue le 8 septembre 2004. Il a confirmé et étendu sa jurisprudence dans des décisions ultérieures comme la décision DEI c. Pays-Bas ${ }^{7}$ du 20 octobre 2009. A l'occasion de cette dernière réclamation, les Etats parties s'étaient positionnés au travers de la résolution adoptée par le Comité des Ministres à la suite de la décision du Comité et par laquelle il « reconnaît les limites du champ d'application personnel de la Charte sociale européenne (révisée), prévues au paragraphe 1 de l'annexe à la Charte" même s'il note que "cela ne décharge pas les Etats de leur responsabilité de prévenir l'état de sans-abri des personnes en situation irrégulière dans leurs juridictions, en particulier lorsqu'il s'agit de mineurs ». Une telle responsabilité ne découlerait donc pas de leur engagement conventionnel.

11 Le refus des Etats parties d'étendre le champ d'application de la Charte aux étrangers s'est encore illustré récemment par leur réponse négative ou leur absence de réponse à une sollicitation du Comité européen des droits sociaux en ce sens. Celui-ci a en effet envoyé le 13 juillet 2011 un courrier incitant les Etats parties à faire une déclaration par laquelle ils étendraient les droits ou certains droits de la Charte à toute personne relevant de leur juridiction. Il semble qu'à ce jour, aucun Etat n'a présenté une telle déclaration et deux Etats, la Lituanie et les Pays-Bas, ont formellement décliné l'invitation ${ }^{8}$. La clarté des termes de la Charte et de la volonté des Etats parties n'a pas empêché le Comité européen de confirmer sa jurisprudence en l'espèce et d'admettre l'applicabilité des articles $13 \$ 4$ et $31 \S 2$ de la Charte aux migrants quelles que soient leur nationalité et leur statut juridique (FEANTSA c. Pays-Bas, $\S \S 61$ et $142 ;$ CEC c. Pays-Bas, $\S \S 75$ et 130).

\section{B - Une extension cantonnée aux droits les plus fondamentaux de la personne}

12 Afin de justifier l'applicabilité de la Charte sociale européenne à l'ensemble des étrangers, le Comité européen des droits sociaux développe un raisonnement fondé sur la dignité de la personne humaine qui lui permet d'étendre la protection de la Charte s'agissant d'un socle restreint de droits considérés comme les plus fondamentaux.

13 A la lecture de la jurisprudence du Comité européen des droits sociaux, l'extension du champ d'application de la Charte aux étrangers semble découler d'une interprétation dynamique, téléologique et systémique de ce traité. Il rappelle ainsi que «l'objet et le but de la Charte, instrument de protection des droits de l'homme, consistent à protéger des droits non pas théoriques et illusoires mais concrets et effectifs ${ }^{9}$. La nature de traité de droits de l'homme ainsi que le principe d'effectivité de ces droits commandent donc d'interpréter strictement les restrictions qui leur sont apportées ${ }^{10}$. Par ailleurs, la Charte doit être lue à la lumière des autres instruments du droit international ${ }^{11}$. A ce titre, le fait que la grande majorité des traités de consécration de droits de l'homme s'adressent à toute personne sous la juridiction des Etats parties peut s'avérer pertinent, en particulier le fait que le Pacte international relatif aux droits économiques, sociaux et culturels (PIDESC), cité 
comme droit pertinent dans les décisions qui nous intéressent, ne connaisse pas une telle restriction de son champ d'application personnel.

Cela étant, la référence au principe de dignité humaine apparaît comme le principal pilier du raisonnement du Comité. Présenté comme la « valeur fondamentale au cœur du droit européen des droits de l'homme » dont la Charte ${ }^{12}$, ce principe interdit que des individus soient laissés dans une situation qui serait contraire au respect de leur dignité. Le Comité considère ainsi, qu'à titre exceptionnel, dans le cas où la préservation de la dignité des personnes est en jeu, certains droits de la Charte peuvent être applicables à toute personne relevant de la juridiction de l'Etat.

Dans sa décision FEANTSA c. Pays-Bas, le Comité synthétise les principes de son interprétation qui permettent de déterminer les droits devant être garantis: «la restriction du champ d'application personnel de la Charte figurant dans l'Annexe ne saurait se prêter à une interprétation qui aurait pour effet de priver les étrangers en situation irrégulière de la protection des droits les plus élémentaires consacrés par la Charte, aussi bien que de porter préjudice à leurs droits fondamentaux, tels que le droit à la vie ou à l'intégrité physique, ou encore le droit à la dignité humaine. Une telle application aux migrants en situation irrégulière serait justifiée dans le seul cas où leur exclusion de la protection assurée par les dispositions de la Charte aurait des conséquences préjudicielles graves pour leurs droits fondamentaux et créerait, par conséquent, à l'encontre des étrangers en question une situation inacceptable dans la jouissance de ces droits, par rapport à la situation des nationaux et des étrangers en séjour régulier 》 (\$58).

Une telle interprétation de la Charte lui avait permis antérieurement d'exiger le bénéfice pour les mineurs en situation irrégulière du droit à la protection de la santé ${ }^{13}$, du droit à la protection sociale, juridique et économique ${ }^{14}$ et à l'hébergement ${ }^{15}$.

17 Pour ce qui concerne l'ensemble des étrangers même en situation irrégulière, le Comité avait affirmé l'applicabilité de l'article $13 \$ 4$ dans la mesure où cette disposition exige la fourniture d'une assistance médicale d'urgence ${ }^{16}$ ainsi que celle de l'article $31 \$ 2$ dans la mesure où ces personnes ne peuvent se retrouver privées de tout abri ${ }^{17}$.

Dans les décisions analysées, le Comité considère que l'ensemble des migrants peuvent se prévaloir d'un droit à l'assistance sociale et médicale d'urgence (FEANTSA c. Pays-Bas, § 142 ; CEC c. Pays-Bas, § 75). L'assistance ne peut donc être uniquement médicale, ce qui est affirmé pour la première fois à l'occasion d'une réclamation collective, même si le Comité l'avait déjà fait lors de l'examen des rapports nationaux ${ }^{18}$. Par ailleurs, l'applicabilité de l'article $31 \$ 2$ à l'ensemble des étrangers quel que soit leur statut juridique est admise ( FEANTSA c. Pays-Bas, § $61 ;$ CEC c. Pays-Bas, § 130), c'est-à-dire leur droit à se voir offrir une solution d'hébergement, là encore de manière inédite dans la mesure où ce droit ne bénéficie pas seulement aux enfants.

On le voit, sont concernés par l'exception à la restriction ratione personae, les droits les plus fondamentaux des individus qui affectent directement leur intégrité physique, c'està-dire les dispositions du traité qui offrent un droit à une assistance sociale et médicale d'urgence y compris le droit à un abri. Le Comité se refuse pour l'heure à aller au-delà dans la remise en cause de la lettre du traité. Il a ainsi rejeté l'applicabilité de l'article $31 \$ 1$ qui garantit le droit à un logement d'un niveau suffisant ${ }^{19}$ ainsi que celle de l'article 30 qui consacre le droit à la protection contre la pauvreté et l'exclusion sociale ${ }^{20}$. Au fur et à mesure de ses décisions, le Comité contribue donc à définir une sorte de socle minimal de droits fondamentaux qui doivent bénéficier à toute personne, sans 
exclusion, au nom du respect de la dignité humaine. Pour ce qui concerne ce noyau dur de droits sociaux, les Etats ne peuvent donc écarter les étrangers de leur bénéfice que ce soit par leur législation ou pratiques nationales ou par leurs engagements conventionnels internationaux. Les décisions du Comité permettent également de mieux cerner le contenu matériel des droits auxquels les étrangers peuvent prétendre.

\section{$2 \%$ - L'étendue du droit à une assistance sociale d'urgence des étrangers en situation irrégulière}

Dans la mesure où la dignité de tout être humain est en jeu, le Comité considère que la Charte garantit un droit subjectif à la satisfaction des besoins élémentaires (A) que l'Etat se doit de respecter sans justification possible de sa défaillance (B).

\section{A - Un droit subjectif à la satisfaction des besoins matériels élémentaires}

De la même manière que pour déterminer l'applicabilité de la Charte, le principe de dignité de la personne humaine est invoqué par le Comité européen des droits sociaux pour contrôler l'application des dispositions de la Charte. Il considère ainsi que ce principe commande le droit de se voir fournir, dans une situation d'urgence, un hébergement ainsi que les biens matériels nécessaires à sa subsistance. En effet, sous l'angle de l'article 31\$2 (Réduction de l'état de sans-abri), le Comité rappelle que «le droit à l'hébergement est étroitement lié au droit à la vie et crucial pour le respect de la dignité humaine de chaque individu » (CEC c. Pays-Bas, §137.). De surcroît, au nom de la dignité des personnes hébergées, l'abri doit répondre "aux exigences de sûreté, de santé et d'hygiène et, en particulier, disposer des éléments de confort essentiels tels que l'accès à l'eau, ainsi qu'un chauffage et un éclairage suffisants. Une autre exigence fondamentale est la sûreté des alentours immédiats " (FEANTSA c. Pays-Bas, § $108 ;$;EC c. Pays-Bas, § 138).

Par ailleurs, les Etats n'étant pas contraints par leurs obligations internationales de proposer des solutions de relogement aux étrangers en situation irrégulière, l'expulsion de ces derniers de leur lieu d'hébergement doit être prohibée dans la mesure où «elle place les intéressés, en particulier les enfants, dans une situation d'extrême détresse, qui est contraire au respect de la dignité humaine » (CEC c. Pays-Bas, § 141 et FEANTSA c. Pays-Bas, § 110).

Sur le fondement de l'article $13 \$ 4$ (Assistance d'urgence spécifique aux non-résidents) de la Charte, les deux décisions affirment l'obligation des Etats parties de fournir une assistance aux personnes concernées en vue de répondre à des besoins urgents et importants : "hébergement, nourriture, soins médicaux d'urgence et vêtements " (FEANTSA c. Pays-Bas, \$171 et CEC c. Pays-Bas, \$105). Cette assistance doit être accordée sans condition que ce soit de résidence (Ibidem), de régularité de séjour (CEC c. Pays-Bas, $\$ \S 73$ et 108; FEANTSA c. Pays-Bas, § 183), ou de coopération dans l'organisation de sa propre expulsion ( CEC c. Pays-Bas, § 107). En effet, refuser une telle assistance fait « indéniablement » courir 
aux personnes concernées «le risque de subir des dommages graves irréparables pour leur vie et leur dignité humaine » (CEC c. Pays-Bas, § 122).

Afin de conforter son raisonnement, le Comité se réfère aux jurisprudences d'autres organes et juges internationaux et européens au travers desquelles se dessine un droit à une assistance minimale pour tous. Il évoque notamment des observations générales du Comité des droits économiques, sociaux et culturels des Nations-Unies qui, interprétant notamment l'article 11 du PIDESC ${ }^{21}$, considèrent que les Etats ont des obligations " indissociables de la dignité de la personne humaine » auxquelles ils ne peuvent déroger et qui " recouvrent l'accès pour tous à un abri et aux aliments de base, indépendamment de leur statut de résidence ${ }^{22}$. De manière moins convaincante, le CEDS s'appuie également sur la Convention européenne des droits de l'homme et le droit de l'Union européenne.

S'agissant de la Convention, et comme cela est rappelé (CEC c. Pays-Bas, §§ 24-29), elle ne consacre aucun droit pour les étrangers en situation irrégulière de se voir fournir un logement, ni de recevoir une assistance financière ou de demeurer sur le territoire d'un Etat membre pour recevoir des soins médicaux. Le CEDS évoque le fait que la Cour n'ait pas exclu la possibilité d'une violation de l'article 3 CEDH s'agissant d'« un requérant totalement dépendant de l'aide publique serait confronté à l'indifférence des autorités alors qu'il se trouverait dans une situation de privation ou de manque à ce point grave qu'elle serait incompatible avec la dignité humaine ", ce qu'elle a affirmé dans l'arrêt M.S.S. c. Belgique et Grèce $^{23}$. Toutefois, la Cour n'a jamais estimé que le seuil de gravité nécessaire à la reconnaissance d'un traitement inhumain et dégradant avait été atteint lorsque des personnes en difficultés l'en ont saisi ${ }^{24}$.

Certes, dans l'arrêt M.S.S., les conditions d'existence du requérant ont constitué une violation de l'article 3, cependant, c'est le statut de demandeur d'asile de celui-ci ainsi -

que les obligations de la Grèce en vertu du droit de l'Union européenne qui ont emporté l'appréciation de la Cour ${ }^{25}$. Il est question ici de la Directive 2003/9/CE ${ }^{26}$ dite Directive « accueil » que le CEDS fait également figurer au titre des textes pertinents du droit de l'Union européenne dans ses décisions. Cette norme européenne impose en effet aux Etats membres, la fourniture de conditions matérielles d'accueil (logement, habillement nourriture et une allocation journalière) pour les demandeurs d'asile.

Le Comité cite l'arrêt Fedasil c. Saciri27 dans lequel la Cour de Justice de l'Union européenne est venue préciser que le principe de dignité s'opposait à ce qu'un demandeur d'asile soit privé même temporairement de la protection des garanties minimales établies par la directive. Cette affirmation n'empêche pas, d'une part, que la directive prévoit des cas dans lesquels les Etats membres sont susceptibles de retirer les conditions d'accueil aux demandeurs d'asile et d'autre part, que ces conditions ne bénéficient pas aux étrangers qui sont eux en situation irrégulière. Le droit de l'Union européenne n'impose aucune obligation en matière d'accueil ou d'assistance des étrangers en situation irrégulière et ce, y compris dans la directive " retour $»^{28}$ qui concerne directement leur situation.

En définitive, les jurisprudences des Cours européennes permettent davantage de conclure à un droit à l'assistance découlant du statut spécifique de demandeur d'asile qu'à un droit universel. L'assistance d'urgence garantie par la Charte sociale européenne complète donc la protection offerte par le droit de l'Union puisqu'elle est octroyée aux étrangers "qui ne remplissent pas, ou ne remplissent plus, les critères " pour bénéficier de cette dernière (CEC c. Pays-Bas, $\S 117$ ). Sont notamment concernés, les demandeurs d'asile qui voient leur demande rejetée. 
29 Le droit à l'assistance garanti sur le fondement de l'article 13 est en outre un droit subjectif exigible devant une instance indépendante ${ }^{29}$. La législation nationale doit donc accompagner ce droit d'un recours dont l'application doit être effective en pratique ( FEANTSA c. Pays-Bas, § 187).

30 En l'espèce, l'Etat défendeur est condamné car il n'a pu établir que «que tous les étrangers sans ressources, qu'ils soient en situation régulière ou irrégulière sur le territoire néerlandais, jouissait d'un droit légalement reconnu à la satisfaction de ses besoins matériels élémentaires (nourriture, vêtements, logement) dans les situations d'urgence » (FEANTSA c. Pays-Bas, § 173 et CEC c. Pays-Bas, $§ 108)^{30}$.

31 En revanche, l'accès aux soins médicaux d'urgence étant assuré, la conformité de la situation nationale à cet égard est constatée (CEC c. Pays-Bas, § 125 et FEANTSA c. Pays-Bas, §186).

32 Au regard de cette jurisprudence, le droit français semble en conformité dans la mesure où l'article L. 345-2-2 du Code de l'action sociale et des familles dispose que "Toute personne sans abri en situation de détresse médicale, psychique ou sociale a accès, à tout moment, à un dispositif d'hébergement d'urgence». A ce titre, la situation de la France a été jugée conforme à la Charte dans le cadre de la procédure sur rapports ${ }^{31}$. En outre depuis, le droit à un hébergement d'urgence a été qualifié de liberté fondamentale au sens de l'article L.521-2 du Code de justice administrative et peut donc donner lieu à un référéliberté $^{32}$. La loi DALO ${ }^{33}$ offre également un droit à un hébergement sans condition de régularité s'agissant des centres d'hébergement d'urgence. Le Comité européen des droits sociaux a considéré favorablement ces dispositions dans l'attente d'informations supplémentaires sur les conditions pratiques de cet accueil ${ }^{34}$.

\section{B - Une défaillance de l'Etat injustifiable}

33 En plus de la définition du contenu des obligations étatiques, les deux décisions sur le bien-fondé ont l'intérêt de répondre aux arguments traditionnellement avancés par les Etats pour justifier leur refus d'accorder une assistance sociale aux migrants en situation irrégulière. Trois tentatives de justifications sont ainsi balayées par le Comité: la politique migratoire, la compétence des collectivités locales et la situation économique.

Un premier motif invoqué par le Gouvernement défendeur afin de justifier son comportement est celui de l'exigence de mener à bien sa politique d'immigration. Le raisonnement est connu, les étrangers en situation illégale n'ont pas vocation à demeurer sur le territoire étatique, en conséquence, leur proposer des accommodations, notamment un hébergement, irait à l'encontre de l'objectif d'incitation à un retour rapide vers leur pays d'origine. Le Comité ne nie évidemment pas la légitimité de l'objectif en rappelant que le droit international garantit le droit des Etats « de contrôler l'entrée sur leur territoire, le séjour et l'expulsion des ressortissants étrangers " (FEANTSA c. Pays-Bas, § 180). Cependant, il considère le refus de proposer un hébergement d'urgence aux personnes en situation irrégulière qui le nécessitent comme un moyen disproportionné en vue d'atteindre ce but légitime. Il estime en effet que des moyens moins lourds sont à la disposition de Gouvernement pour réaliser ses objectifs (CEC c. Pays-Bas, §§ 121-123 et FEANTSA c. Pays-Bas, §§ 181-183).

Dans une deuxième série d'arguments, le Gouvernement néerlandais évoque la compétence des municipalités qui est prévue par la loi et le fait qu'elle soient mieux 
placées pour mettre en place les structures d'accueil. Le Comité rappelle sa jurisprudence constante $^{35}$ selon laquelle si les Etats ont le droit de confier des compétences aux collectivités territoriales, ils demeurent les seuls responsables vis-à-vis de leurs obligations internationales. Aussi, ils doivent s'assurer que les collectivités respectent bien les obligations de la Charte et ce, sans qu'une uniformité du droit et des pratiques soient exigées (CEC c. Pays-Bas, § 118; FEANTSA c. Pays-Bas, § 112-113). Les autorités centrales ne peuvent donc invoquer la possibilité qu'ont les collectivités locales de proposer un hébergement d'urgence aux personnes concernées en l'absence de contrainte au niveau national ou le fait que certains organismes privés proposent également leur aide. Elles doivent en effet s'assurer que cette assistance est effectivement fournie à toute personne qui se trouve dans le besoin (CEC c. Pays-Bas, § 119 et FEANTSA $c$. Pays-Bas, §§ 120-125).

Enfin, l'argument économique est immanquablement avancé pour justifier le nombre insuffisant de places en centre d'accueil. En l'espèce, l'argument est invoqué par le Gouvernement défendeur dans la décision FEANTSA c. Pays-Bas afin de justifier l'insuffisance de l'offre de logements sociaux qui doivent bénéficier aux ressortissants de l'Etat membre ou étrangers en situation régulière qui le nécessitent. Même si les étrangers en situation irrégulière ne sont pas directement concernés par l'appréciation du Comité, le raisonnement tenu par celui-ci s'avère très utile dans la situation, malheureusement fréquente, où l'offre d'hébergement d'urgence est également déficiente. Aussi, le Comité ne reçoit pas l'argument de la situation économique dégradée en cette période de crise. Il rappelle ainsi que la «crise économique ne doit pas se traduire par une baisse de la protection des droits reconnus par la Charte et que les Etats parties se doivent dès lors de prendre toutes les mesures nécessaires pour faire en sorte que ces droits soient effectivement garantis au moment où le besoin de protection se fait le plus sentir » (\$128) ce qu'il avait déjà eu l'occasion d'affirmer s'agissant des mesures d'austérité grecques ${ }^{36}$.

Les Etats ne sont donc pas exemptés de leurs obligations. Toutefois s'agissant de la mise en œuvre de politiques sociales spécialement coûteuses comme celle des logements sociaux, le Comité n'impose aux Etats qu'une obligation de moyens renforcée qui exige de l'Etat de prendre toutes les mesures en son pouvoir mais également de justifier d'une volonté réelle d'améliorer progressivement la situation.

S'agissant de l'article 31\$2, la Charte impose ainsi de :

«-mettre en œuvre des moyens (normatifs, financiers, opérationnels) propres à permettre de progresser réellement vers la réalisation des objectifs assignés par la Charte ;

-tenir des statistiques dignes de ce nom permettant de confronter besoins, moyens et résultats ;

-procéder à une vérification régulière de l'effectivité des stratégies arrêtées ; -définir des étapes et ne pas reporter indéfiniment le terme des performances qu'ils se sont assignées ;

-être particulièrement attentifs à l'impact des choix opérés par eux sur l'ensemble des catégories de personnes concernées et singulièrement celles dont la vulnérabilité est la plus grande $»^{37}$.

Le nombre insuffisant de places en structures d'accueil spécifiques pour les groupes vulnérables, en particulier les femmes et les mineurs, constitue également une violation de la Charte sociale européenne. En effet, le Comité rappelle que «les structures d'accueil d'urgence doivent toujours répondre aux conditions de sécurité requises et être adaptés aux besoins des individus qui appartiennent à ces groupes» ( $\S 135)$. Aussi, le fait de rediriger ces 
personnes vers des structures d'accueil général n'est pas conforme aux obligations européennes.

Ces précisions mènent à s'interroger sur la réelle conformité de la situation française aux exigences de la Charte. En effet, si les textes garantissent bien un droit à une assistance sociale d'urgence, les faits traduisent des défaillances dans l'offre d'accueil d'urgence, la plupart des demandes d'hébergement étant refusées faute de places disponibles ${ }^{38}$. Le contrôle général et objectif auquel procède le Comité européen des droits sociaux dans le cadre de la procédure de réclamations collectives aurait ainsi l'intérêt de pouvoir apprécier la politique étatique et ses résultats afin de rechercher l'existence ou non d'améliorations tangibles, ainsi que d'une volonté réelle des autorités en ce sens. Une telle appréciation n'est en effet pas possible dans le cadre de recours individuels. De tels recours ont cependant l'intérêt, par leur multiplication, d'inciter les autorités à adopter des mesures positives.

Toutefois, la pression opérée par le contrôle judiciaire national n'est pas véritablement efficace dans la mesure où le Conseil d'Etat n'impose qu'une obligation de moyens. En effet, « seule une carence caractérisée des autorités de l'État » est sanctionnée par le juge à qui il « incombe d'apprécier dans chaque cas les diligences accomplies par l'administration en tenant compte des moyens dont elle dispose ainsi que de l'âge, de l'état de santé et de la situation de famille de la personne intéressée $"^{39}$. La saturation du dispositif d'hébergement est donc un argument toujours accueilli par le juge.

Une seule affaire récente a donné lieu à une appréciation contraire, la Haute juridiction administrative ayant considéré que le manque de place et de budget ne permettait pas d'exonérer l'Etat de sa responsabilité concernant un mineur étranger isolé ${ }^{40}$. Comme cela a été observé en matière d'accueil des demandeurs d'asile ${ }^{41}$, le manque structurel de places d'hébergement conduit à une priorisation parmi les personnes en détresse qui consiste en définitive à n'octroyer une assistance minimale qu'aux personnes les plus vulnérables. Or, le Comité rappelle que cette assistance est due à toute personne qui le nécessite.

CEDS, $1^{\mathrm{er}}$ juillet 2014, Conférence des Eglises européennes (CEC) c. Pays-Bas, $\mathrm{n}^{\circ}$ 90/2013

CEDS, 2 juillet 2014, Fédération européenne des Associations nationales travaillant avec les Sans-abri (FEANTSA) c. Pays Bas, $\mathrm{n}^{\circ}$ 86/2012

Les Lettres « Actualités Droits-Libertés » (ADL) du CREDOF (pour s'y abonner) sont accessibles sur le site de la Revue des Droits de l'Homme (RevDH) - Contact 


\section{NOTES}

1. La CEC est une organisation internationale non gouvernementale dotée du statut participatif au Conseil de l'Europe et donc habilitée à saisir le Comité d'une réclamation collective.

2. CEDS, 25 octobre 2013, CEC c. Pays-Bas, réclam. n90/2013 (mesures immédiates) ; CEDS, 25 octobre 2013, FEANTSA c. Pays Bas, réclam. n86/2012 (mesures immédiates).

3. « Article 31 - Droit au logement

En vue d'assurer l'exercice effectif $d u$ droit au logement, les Parties s'engagent à prendre des mesures destinées:

2. à prévenir et à réduire l'état de sans-abri en vue de son élimination progressive; »

4. «Article 13 - Droit à l'assistance sociale et médicale

En vue d'assurer l'exercice effectif du droit à l'assistance sociale et médicale, les Parties s'engagent:

1. à veiller à ce que toute personne qui ne dispose pas de ressources suffisantes et qui n'est pas en mesure de se procurer celles-ci par ses propres moyens ou de les recevoir d'une autre source, notamment par des prestations résultant d'un régime de sécurité sociale, puisse obtenir une assistance appropriée et, en cas de maladie, les soins nécessités par son état;

2. à veiller à ce que les personnes bénéficiant d'une telle assistance ne souffrent pas, pour cette raison, d'une diminution de leurs droits politiques ou sociaux;

3. à prévoir que chacun puisse obtenir, par des services compétents de caractère public ou privé, tous conseils et toute aide personnelle nécessaires pour prévenir, abolir ou alléger l'état de besoin d'ordre personnel et d'ordre familial;

4. à appliquer les dispositions visées aux paragraphes 1, 2 et 3 du présent article, sur un pied d'égalité avec leurs nationaux, aux ressortissants des autres Parties se trouvant légalement sur leur territoire, conformément aux obligations qu'elles assument en vertu de la Convention européenne d'assistance sociale et médicale, signée à Paris le 11 décembre 1953 ».

5. K. MICHELET, Les droits sociaux des étrangers, L'Harmattan, 2003, 498p ; D. ROMAN (dir.), Les droits sociaux, entre droits de l'Homme et politiques sociales. Quels titulaires pour quels droits?, LGDJ, 2012, 200p.

6. CEDS, 8 septembre 2004, FIDH c. France, réclam. n 14/2003.

7. CEDS, 20 octobre 2009, DEI c. Pays-Bas, réclam. n 47/2008.

8. CEDS, Conclusions 2011, Introduction générale, §§21-23.

9. CEDS, 9 septembre 1999, CIJ c. Portugal, réclamation $n^{\circ} 1 / 1998, \S 32$; FEANTSA c. Pays-Bas, $\S$ 224.

10. CEDS, DEI c. Pays-Bas, op. cit., § 36 .

11. CEDS, DEI c. Pays-Bas, op. cit., §29 ; FIDH c. France, op. cit., §26 ; CEC c. Pays-Bas, §§ 68-69.

12. CEDS, FIDH c. France, op. cit., $\S 31$; CEC c. Pays-Bas, $\S 115$.

13. CEDS, 23 octobre 2012, DEI c. Belgique, réclam. $n^{\circ} 69 / 2011, \S 102$.

14. Ibid., $\S \S 39$ et 86 .

15. CEDS, DEI c. Pays-Bas, op. cit., $\S \S 47-48$ et 64.

16. CEDS, FIDH c. France, op. cit., $\$ 32$.

17. CEDS, DEI c. Pays-Bas, op. cit., § 63 ; DEI c. Belgique, op. cit., \$136.

18. Voir entre autres, CEDS, Conclusions 2009, Italie, article $13 \$ 4$.

19. CEDS, DEI c. Pays-Bas, op. cit., $§ 45$.

20. CEDS, DEI c. Belgique, op. cit., § 147 ; FEANTSA c. Pays-Bas, § 211. 
21. «1. Les Etats parties au présent Pacte reconnaissent le droit de toute personne à un niveau de vie suffisant pour elle-même et sa famille, y compris une nourriture, un vêtement et un logement suffisants, ainsi qu'à une amélioration constante de ses conditions d'existence ".

22. CEDS, CEC c. Pays-Bas, \$§113-114. La décision se réfère à l'Observation générale $n^{\circ} 12$, Le droit a une nourriture suffisante (article 11), E/C.12/1999/5, §§4, 15 ; à l'Observation générale $n^{\circ} 4$, Le droit à un logement suffisant (art. 11§1), E/1992/23, §§6-7 et à l'observation générale $\mathrm{n}^{\circ} 14$, Le droit au meilleur état de santé susceptible d'être atteint, E/C.12/2000/4, §§43, 47.

23. CEDH, GC, 21 janvier 2011, M.S.S. c. Belgique et Grèce, nº 30696/09, § 253.

24. CEDH, déc., 28 octobre 1999, Pančenko c. Lettonie, req. 40772/98; déc., 26 juin 2001, O’Rourke c. Royaume-Uni, req. 39022/97 ; déc., 23 avril 2002, Larioshina c. Russie, req. 56869/00 ; déc., 18 juin 2009, Budina c. Russie, req. 45603/05.

25. CEDH, MSS c. Belgique et Grèce, op. cit., §§ 251 et 263.

26. Cette directive sera remplacée par la Directive 2013/33 établissant des normes pour l'accueil des personnes demandant la protection internationale (refonte) à partir du 21 juillet 2015.

27. CJUE, 27 février 2014, affaire C-79/13 ; Marie-Laure Basilien-Gainche et Serge Slama, « Implications concrètes $\mathrm{du}$ droit des demandeurs d'asile aux conditions matérielles d'accueil dignes ", La Revue des droits de l'homme, 5 mars 2014.

28. Directive 2008/115 du 16 décembre 2008 relative aux normes et procédures communes applicables dans les Etats membres au retour des ressortissants de pays tiers en séjour irrégulier. 29. CEDS, FEANTSA c. Pays-Bas, § 169 ; Conclusions I, 1969, Observation interprétative.

30. Le Comité avait déjà constaté l'inconventionnalité de la situation nationale dans ses Conclusions 2009, Pays-Bas, $13 \S 4$ et Conclusions 2013, Pays-Bas, $13 \$ 4$.

31. CEDS, Conclusions 2009 France, article $13 \$ 4$.

32. CE, réf., 10 février 2012, req. $\mathrm{n}^{\circ} 356456$.

33. Article L.441-2-3 du Code de la construction et de l'habitation.

34. CEDS, Conclusions 2011, France, $31 \S 2$.

35. Voir entre autres, CEDS, 8 décembre 2004, CEDR c. Grèce, réclamation $n^{\circ} 15 / 2003, \$ 29 ; 4$ décembre 2012, The Central Association of Carers in Finland c. Finlande, réclamation $n^{\circ} 70 / 2011$, $\S \S 55-56$; 18 mars 2013, FIDH c. Belgique, réclamation nº 75/2011, §54.

36. Voir Lettres ADL du 16 novembre 2012 et du 12 mai 2013.

37. CEDS, FEANTSA, c. Pays-Bas, § 111 et CEDS, 5 décembre 2007, Mouvement international ATD Quart Monde c. France, réclam. $n^{\circ} 33 / 2006, \$ 60$.

38. Voir pour des statistiques récentes le baromètre 115 pour la période hivernale 2013-2014 réalisé par la Fédération nationale des associations d'accueil et de réinsertion sociale.

39. CE, réf., 10 février 2012, op. cit.

40. CE, réf., 12 mars $2014, n^{\circ} 375956$.

41. Marie-Laure Basilien-Gainche et Serge Slama, "Implications concrètes du droit des demandeurs d'asile aux conditions matérielles d'accueil dignes ", La Revue des droits de l'homme, 5 mars 2014. 


\section{RÉSUMÉS}

Par deux décisions sur le bien-fondé, CEC c. Pays-Bas et FEANTSA c. Pays-Bas, rendues les 1er et 2 juillet 2014, le Comité européen des droits sociaux rappelle et précise sa jurisprudence relative aux droits des étrangers en situation irrégulière. Dépassant la limitation du champ d'application personnel prévue par les termes du traité, il considère que toute personne doit bénéficier de certains droits de la Charte sociale européenne au nom du respect de la dignité humaine. Il constate ainsi l'inconventionnalité de la situation aux Pays-Bas en ce que les migrants en situation irrégulière se voient refuser, en droit et en pratique, l'accès à une assistance sociale d'urgence, notamment à une solution d'hébergement.

\section{AUTEUR}

\section{CAROLE NIVARD}

Maître de conférences en droit public à l'Université de Rouen (Centre universitaire rouennais d'études juridiques) 